\title{
Análisis del cambio repentino a docencia remota por la COVID-19 en los resultados de aprendizaje: caso de dos asignaturas anuales básicas en Grados de Ingeniería
}

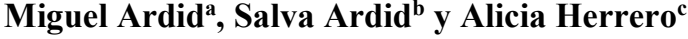 \\ a Departament de Física Aplicada, Universitat Politècnica de València, mardid@fis.upv.es, ${ }^{\mathrm{b}}$ Departament de Física \\ Aplicada, Universitat Politècnica de València, sardid@upv.es y ${ }^{\mathrm{c}}$ Departament de Matemàtica Aplicada, Universitat \\ Politècnica de València, aherrero@mat.upv.es.
}

\section{\$EWBDFW}

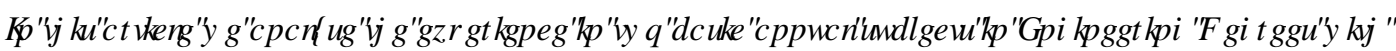

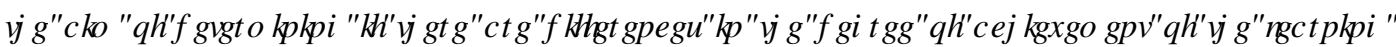

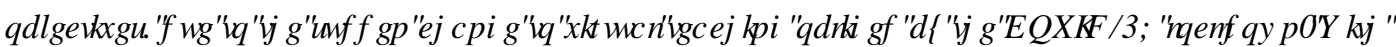

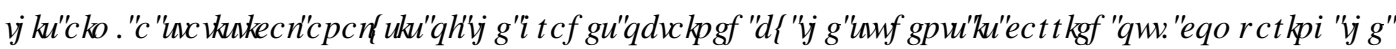

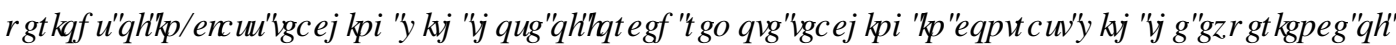

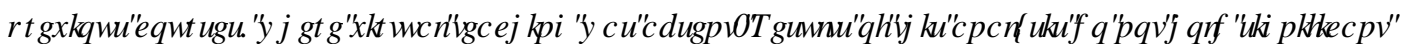

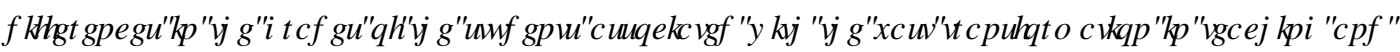

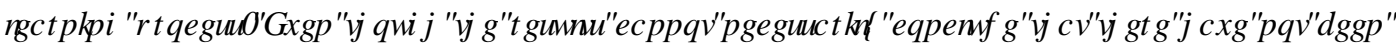
FKDQJ HUIQ

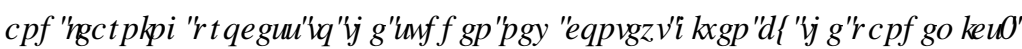

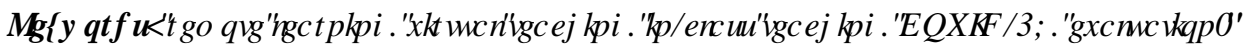

\section{HXP HQ}

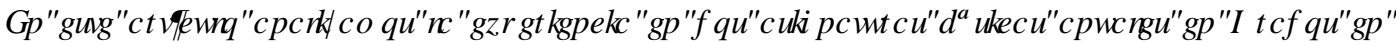

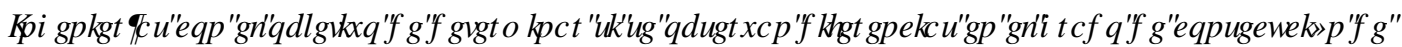

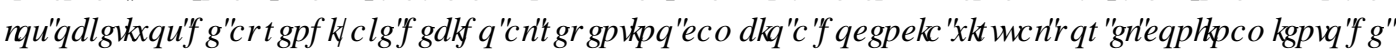

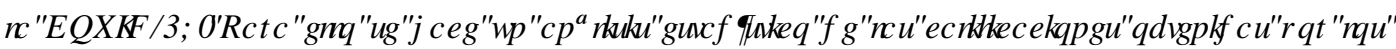

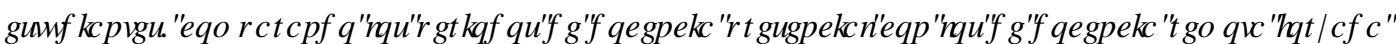

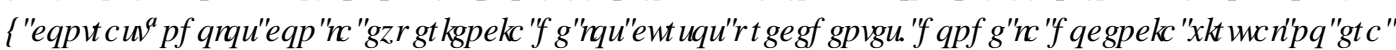

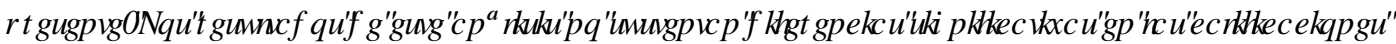

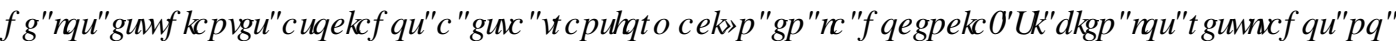

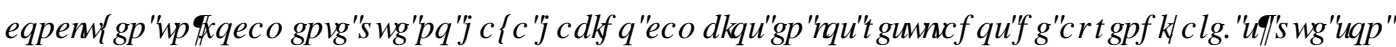

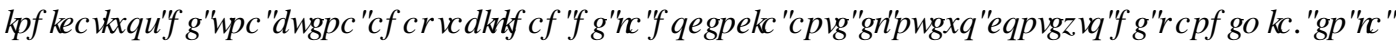

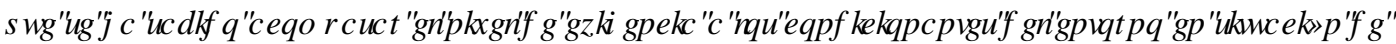
FUMVIRELHHPIGDII

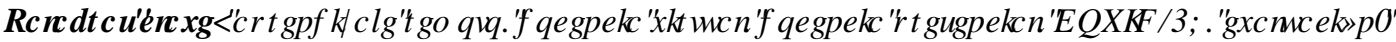

\section{Introducción}

La pandemia mundial de la COVID-19 ha trastocado el normal trascurso de la docencia universitaria en la mayoría de los países, impactando tanto en el modelo docente (Rapanta 2020). como en el rol y situación 
de profesores (Moorhouse, 2020) (Marek, 2021) y estudiantes (Aristovnik 2020). En España, el confinamiento obligó a la transformación urgente de las clases presenciales a un formato online. La percepción generalizada es que ésta se ha realizado de forma bastante aceptable en términos generales, a pesar de la planificación para impartir las diferentes asignaturas en un entorno presencial y la premura del cambio a una metodología completamente online sin tiempo para una planificación concienzuda. Sin embargo, esta percepción no ha sido fundamentada con una sistemática recogida de datos y análisis de los mismos. Uno de los aspectos más delicados en esta transición ha sido la evaluación online masiva, aspecto totalmente novedoso en el ámbito institucional de la mayoría de las Universidades (García-Peñalvo, 2020).

En este artículo analizamos la experiencia en dos asignaturas anuales de Grado en Ingenierías de la Universitat Politècnica de València con el fin de determinar si se observan diferencias en el grado de consecución de los objetivos de aprendizaje, contrastando los periodos de docencia presencial con los de docencia remota forzada. Para ello se hace un análisis estadístico de las calificaciones obtenidas por los estudiantes, comparándolos con la experiencia de los cursos precedentes.

El artículo se organiza de la siguiente forma: en la sección 2 se presentan los objetivos del estudio, en la sección 3 se describe la metodología utilizada. En la sección 4 se detallan los resultados. Finalmente, en la sección 5 se destacan las conclusiones del estudio.

\section{Objetivos}

El objetivo principal de este trabajo es analizar el impacto del cambio súbito de docencia presencial a remota en asignaturas básicas anuales en Grados de Ingeniería. La situación de crisis sanitaria debida a la pandemia de la COVID-19 obligó a estos cambios metodológicos de forma repentina en marzo de 2020. Otro de los objetivos es la comparación entre docencia presencial y virtual, así como la agilidad de la comunidad universitaria, tanto de profesores como de estudiantes, para adaptarse de docencia presencial a remota por causa sobrevenida. Estos objetivos se enmarcan dentro del estudio de dos casos específicos de asignaturas básicas en Grados de Ingeniería.

\section{Desarrollo de la innovación}

Con el fin de evaluar el impacto del cambio de docencia presencial a docencia virtual, se ha procedido al análisis de los resultados de evaluación en dos casos concretos de asignaturas anuales básicas de Grados de Ingeniería que, de forma repentina debido a la pandemia, pasaron de impartirse de forma presencial a virtual : Física del Grado de Ingeniería Aeroespacial y Matemáticas I del Grado de Ingeniería Electrónica y Automática, ambas impartidas en la Escuela Técnica Superior de Ingeniería del Diseño en la Universitat Politècnica de València. Consideramos que, si bien se trata de dos asignaturas básicas de Grado, sus diferencias (principalmente en términos de materia y Grado Universitario) nos permiten estimar el impacto del cambio de docencia en, al menos, asignaturas de los primeros cursos de Ingeniería. Además, otra diferencia fundamental entre los dos casos se establece en términos del número y perfil de los estudiantes: si bien la asignatura de Matemáticas I se enmarca dentro de un grupo reducido de estudiantes con Alto Rendimiento Académico, el análisis de la asignatura de Física se establece a partir de los datos de dos grupos numerosos de estudiantes. El número de estudiantes que completaron la evaluación para cada curso se presenta en la Tabla 1. 


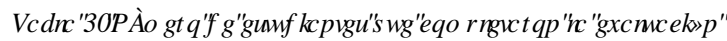

\begin{tabular}{ccccc}
\hline Asignatura & $\mathbf{2 0 1 9 / 2 0}$ & $\mathbf{2 0 1 8 / 1 9}$ & $\mathbf{2 0 1 7 / 1 8}$ & $\mathbf{2 0 1 6 / 1 7}$ \\
\hline Física & 110 & 115 & 119 & 116 \\
Matemáticas & 20 & 19 & 20 & 18 \\
\hline
\end{tabular}

Por otro lado, ambas asignaturas comparten una estructura básica que incluye clases de teoría, problemas, prácticas de laboratorio, y tareas presenciales y no presenciales, algunas de éstas realizadas y evaluadas por equipos, y otras de forma individual. La Tabla 2 desglosa la evaluación de cada una de estas secciones para cada asignatura con su ponderación en la evaluación.

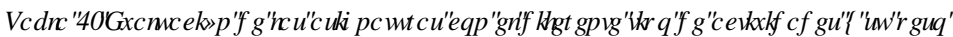

\begin{tabular}{cccc}
\hline Asignatura & Exámenes escritos & $\begin{array}{c}\text { Prácticas de } \\
\text { Laboratorio }\end{array}$ & Tareas \\
\hline Física & Problemas y tests & Memorias & Variadas \\
& (Individual, 70\%) & (Equipo, 20\%) & (individual y por equipos, 10\%) \\
\hline Matemáticas I & Problemas y tests & Actividad & Variadas \\
& (Individual, 70\%) & (Individual, 20\%) & (individual y por equipos, 10\%)
\end{tabular}

En este estudio, comparamos los resultados de las evaluaciones del curso 2019/20 con respecto a los tres cursos anteriores para determinar si el repentino cambio a docencia remota y virtual, debido a la pandemia, tuvo un impacto significativo en la calificación de los estudiantes. De haber un efecto, esperamos que éste se refleje de forma más notable en los exámenes escritos, tanto por su mayor peso como naturaleza. Además, al realizarse de forma individual, la variabilidad de la muestra es mayor, siendo el estudio más sensible. El estudio de las otras actividades: prácticas de laboratorio y tareas, muestra una casuística mucho más diversa. Desde hace algún tiempo se han ido implementando acciones, incorporando docencia virtual en tareas (Ardid, 2015), o más recientemente en prácticas de laboratorio (Ardid, 2021). Aunque de gran interés también, su análisis es más complejo al englobar también trabajo en equipo y toda la casuística y se deja para un futuro estudio, esperando recopilar más datos y evidencias para abordar los objetivos con garantías.

Así pues, y en la línea marcada, se ha hecho hincapié en la observación del último parcial de examen escrito, cuya docencia y evaluación fue totalmente online, con el primer examen parcial, con docencia y evaluación totalmente presencial. El análisis de esta diferencia no se puede realizar directamente, pues usualmente se observan diferencias entre los resultados del primer y último parcial que no tienen que ver con el tipo de docencia, como se comprueba al comparar la misma información en cursos anteriores en los que la docencia no cambió. Estas diferencias se pueden deber tanto a la complejidad de los contenidos de cada parcial (que un parcial sea más complejo que el otro), como a la dinámica docente: por un lado, en el primer parcial puede que haya alumnos que aún no se hayan adaptado al entorno universitario o a la asignatura, por ejemplo, porque carezcan de los conocimientos básicos previos que facilitan el aprendizaje, y esto incide en sus calificaciones iniciales. Por otro lado, respecto al último parcial, se puede dar el caso de que algunos estudiantes se relajen o reorienten sus esfuerzos hacia otras asignaturas, si con las calificaciones anteriores estiman que la asignatura está ya prácticamente aprobada. En definitiva, la comparación directa no es apropiada y se utiliza la comparación con los cursos anteriores como mecanismo de control.

(c)) BY-NC-ND 2021, Universitat Politècnica de València

CRQJUHR, Q5 HGHपिए। 
El análisis se realizará principalmente sobre la asignatura de Física, puesto que se trata de una muestra de datos mayor y por tanto estadísticamente más fiable. Tras lo cual, se aplicará la misma metodología a la asignatura de Matemáticas I (con una muestra de datos y perfil del estudiante diferentes), y finalmente se contrastarán los resultados de una y otra asignatura.

\section{Resultados}

Nuestro objetivo es determinar si el repentino cambio de docencia presencial a docencia remota tuvo un impacto en los resultados de aprendizaje de los alumnos. Pragmáticamente, se asimilan los resultados de aprendizaje a los indicadores habituales al efecto, que son las calificaciones obtenidas durante la evaluación de las asignaturas. En este estudio, como hemos comentado, nos centraremos en las pruebas escritas por tratarse de la sección evaluativa con mayor peso (70\%). En primer lugar se hará un estudio de la asignatura de Física. Después se contrastarán los resultados obtenidos de esta asignatura con los que se obtienen de la asignatura de Matemáticas I para analizar la consistencia de los resultados, y así estimar su posible generalidad.

\subsection{Análisis de la asignatura de Física}

Para evaluar las diferencias entre docencia remota y presencial nos centraremos principalmente en los exámenes escritos, que es, a priori, donde se espera el mayor cambio y la mayor complicación en el paso de docencia presencial a remota. Antes de comparar el primer examen parcial, completamente presencial, con el cuarto y último, completamente remoto, necesitamos analizar si ambos parciales eran ya significativamente distintos en cursos impartidos en los años previos al cambio repentino de docencia, pues en ese caso no se podría adjudicar directamente el efecto al cambio de docencia. Para ello, realizamos un ttest de las calificaciones obtenidas en cada uno de los tres cursos anteriores. Al hacerlo, se observan diferencias significativas ( $\mathrm{p}=0,00126$ ) en el curso $2018 / 19$, pero no se observan diferencias significativas $(\mathrm{p}>0,05)$ en los dos cursos previos. Como hemos comentado, la equivalencia entre exámenes parciales no es trivial, dado que, además de las diferencias en contenidos, interviene condicionantes externos en la localización temporal tales como la adaptación al entorno universitario y a la propia asignatura, o la interrelación con otras asignaturas, especialmente al combinar, un mismo curso académico, asignaturas de carácter anual y cuatrimestral. Cambios en estos efectos podrían explicar la diferencia entre el curso 2018/19 y los dos anteriores a este.

El curso de interés, 2019/20, en el cual se produjo el cambio repentino a docencia virtual, no aprecia tampoco diferencias significativas en las calificaciones de los estudiantes entre el primer y último parcial $(\mathrm{p}=0,124 ; \mathrm{t}$-test). En los siguientes apartados analizamos con más detalle la relación entre estos exámenes parciales y las diferencias entre ellos durante los últimos cursos con el fin de profundizar en los posibles efectos del confinamiento y docencia virtual durante el curso 2019/20. También trataremos de entender posibles causas de estos resultados iniciales: (i) a qué se debe la discrepancia del curso académico 2018/19, y especialmente, (ii) si la ausencia de efecto en el año de interés 2019/20 es debida a que la docencia virtual, en comparación con la presencial no afecta a los resultados de aprendizaje, o si, alternativamente, los resultados de aprendizaje, conjuntamente con la metodología, se han reajustado para adaptarse al contexto de pandemia mundial y confinamiento vivido.

Para profundizar en el estudio, en la Figura 1 se muestra un diagrama de dispersión de la nota del último examen (eje Y) en función de la nota del primer examen (Eje X). Aunque con bastante dispersión, se puede apreciar que existe una correlación entre ambas notas, tal y como cabía esperar. El coeficiente de 
correlación Pearson se incluye en la Tabla 3, conjuntamente con otros parámetros estadísticos de estas variables y sus combinaciones principales. Como se puede observar, existe una tendencia lineal entre ambas notas, si bien el grado de predicción es relativo con una incertidumbre en la estimación de entre 1,8 y 2 puntos. Así pues, atendiendo tanto a la pendiente del ajuste, como a la incertidumbre en la estimación no se aprecia ninguna diferencia significativa para el curso en el que se produjo el confinamiento, 2019/20.

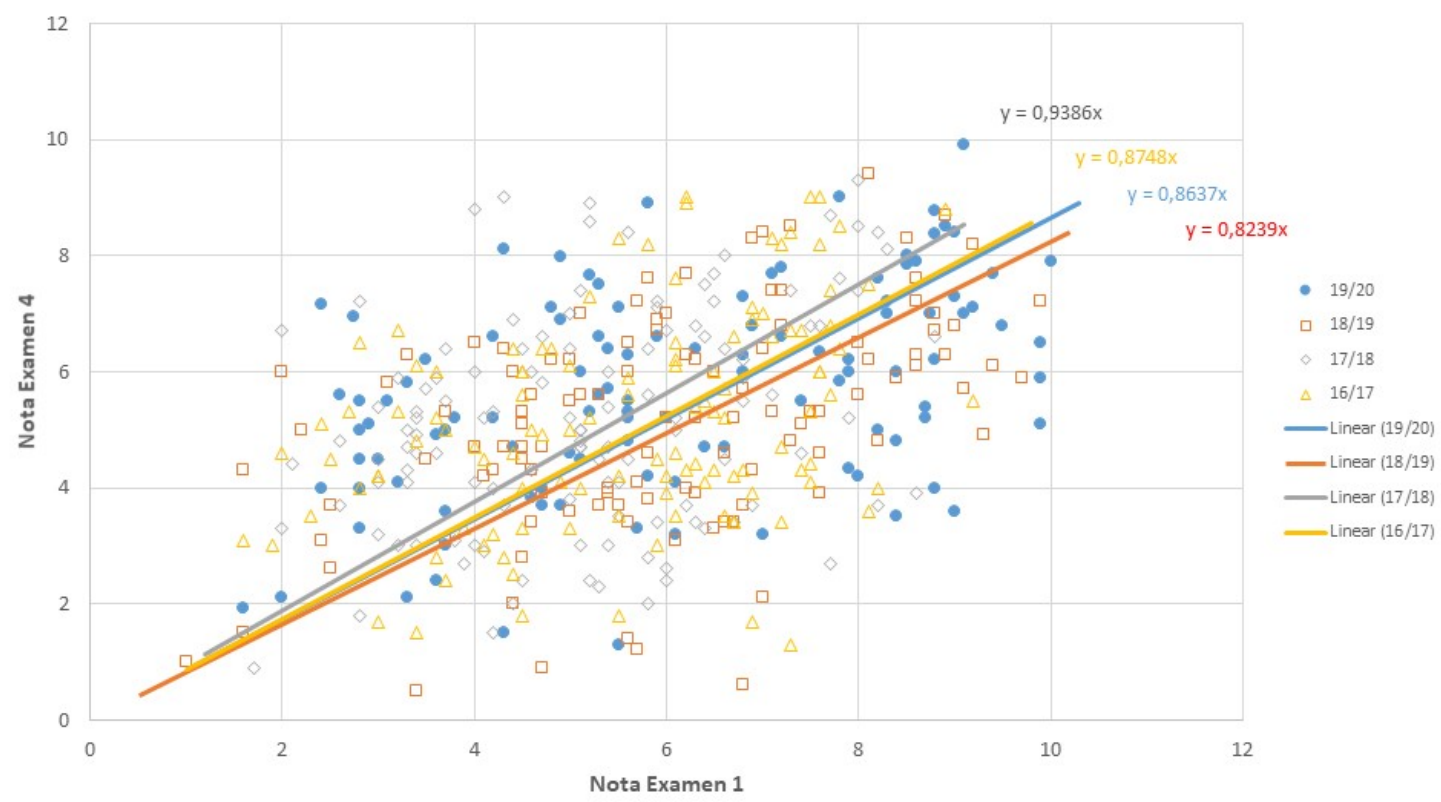

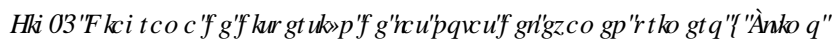

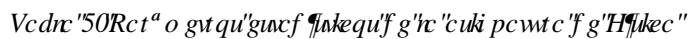

\begin{tabular}{ccccc}
\hline Magnitud & $\mathbf{2 0 1 9 / 2 0}$ & $\mathbf{2 0 1 8} / \mathbf{1 9}$ & $\mathbf{2 0 1 7 / 1 8}$ & $\mathbf{2 0 1 6 / \mathbf { 1 }}$ \\
& & & & $\mathbf{7}$ \\
\hline N & 110 & 115 & 119 & 116 \\
Media Examen 1 & 6,1 & 6,0 & 5,2 & 5,6 \\
Desv. Estándar Ex1 & 2,2 & 1,9 & 1,6 & 1,7 \\
Media Examen 4 & 5,7 & 5,2 & 5,1 & 5,1 \\
Desv. Estándar Ex4 & 1,9 & 1,8 & 1,9 & 1,8 \\
Coef. Correlación & $0,46^{* *}$ & $0,47^{* *}$ & $0,33^{* *}$ & $0,37^{* *}$ \\
(** indica p $<0.001)$ & & & & 5,4 \\
Media Semisuma & 5,9 & 5,6 & 5,2 & 1,5 \\
Desv. Est. Semisuma & 1,7 & 1,6 & 1,4 & 0,22 \\
Media Semidiferencia & 0,21 & 0,41 & 0,013 & 1,0 \\
Desv. Est. Semidif. & 1,1 & 1,0 & 1,0 & \\
\hline
\end{tabular}


Puede resultar incluso más esclarecedor estudiar directamente la diferencia entre los exámenes primero y último y compararlo directamente con cursos anteriores. Por ejemplo, en la Figura 2 se compara la semidiferencia versus la semisuma para los dos últimos cursos. Este tipo de gráficos puede ayudar a apreciar regiones de especial relevancia, clústeres, o sesgos. Sin embargo, al comparar ambos cursos, la distribución es muy parecida. También se muestra en la Figura 3 los histogramas de calificaciones de exámenes, 1 (primero) y 4 (último), y su promedio para los dos últimos cursos. También la diferencia de calificaciones entre estos exámenes y la diferencia de la calificación del último examen con respecto a la estimada para el examen 4 a partir de la obtenida en el examen 1, acorde a lo representado en la Figura 1. Usamos la notación $\mathrm{P}(\mathrm{Ex} 1)$ para resaltar que esta predicción del examen 4 es una función del primer examen. De la distribución de calificaciones de los histogramas se puede apreciar que las calificaciones no siguen una distribución normal, si bien se acercan a ella en algunos casos, especialmente cuando se considera el promedio de ambas. En cambio, las diferencias sí distan mucho de la distribución normal en todos los casos. La distribución resultante es más plana, tanto en su diferencia directa, como en la desviación con respecto a la predicción. Así pues, no se observa un patrón definido, ni una diferencia clara entre ambos cursos y, por tanto, de las gráficas resulta difícil estimar si las diferencias son significativas.

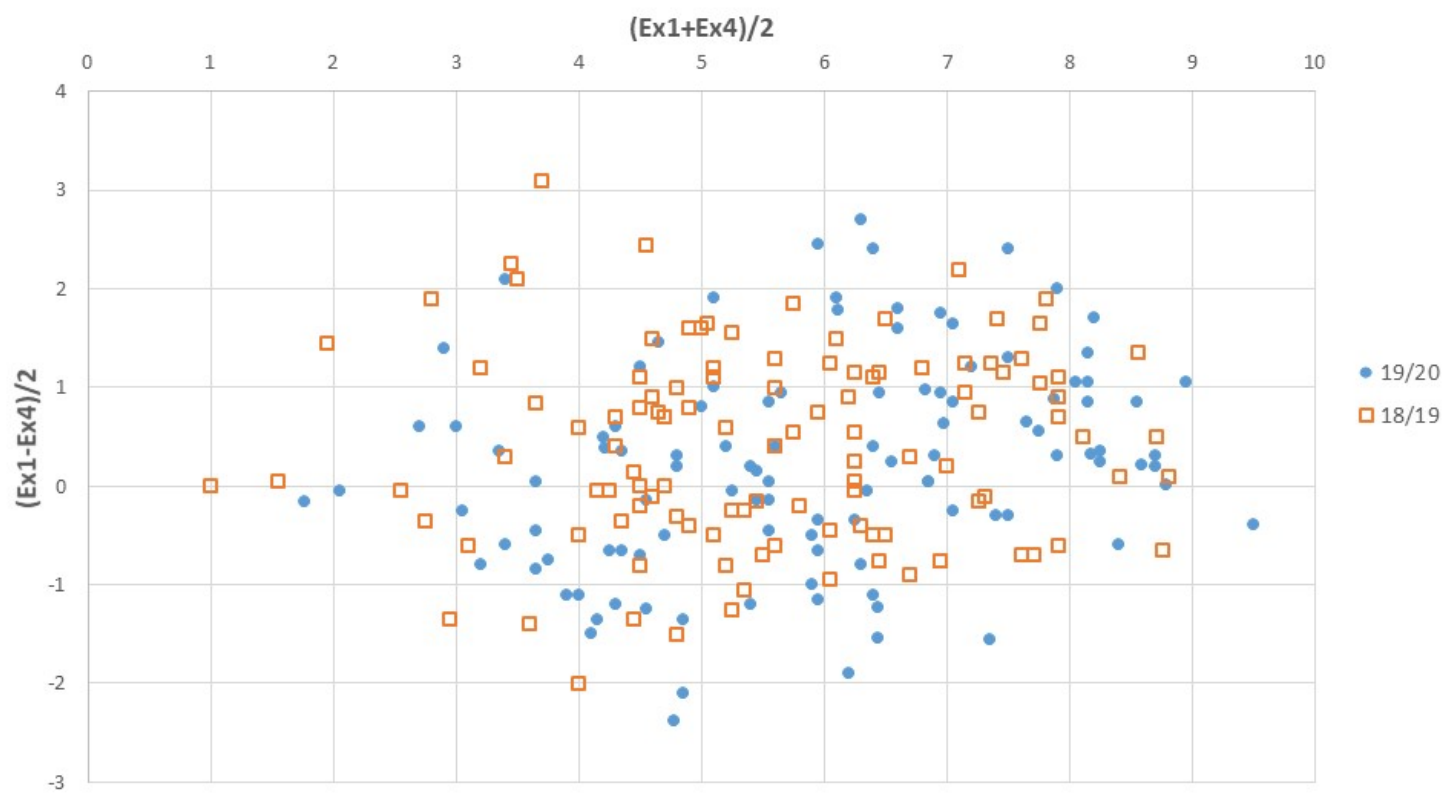

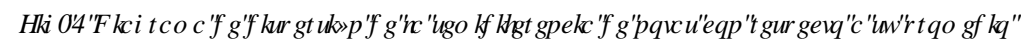



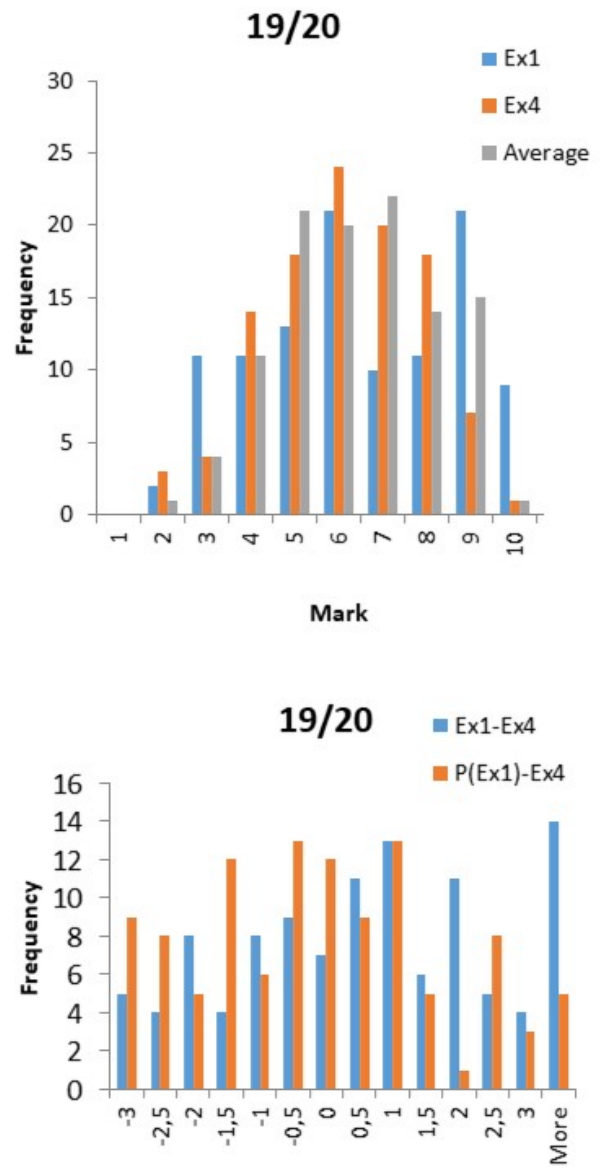

$18 / 19$
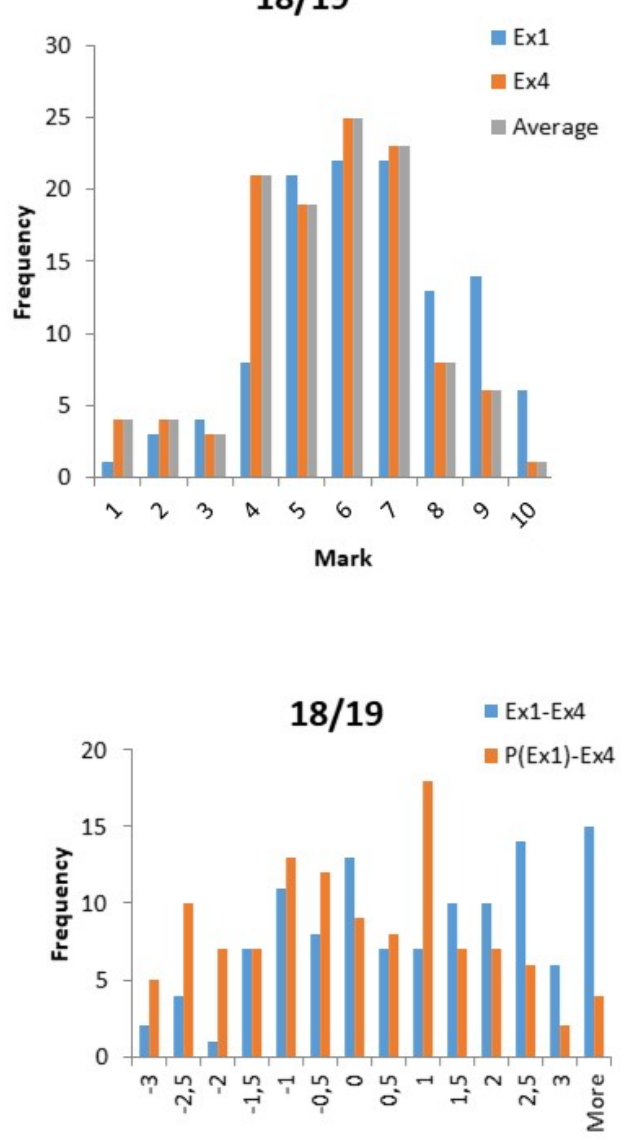

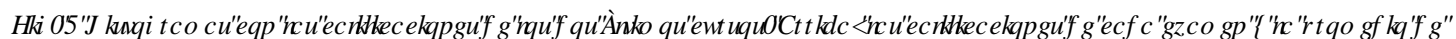

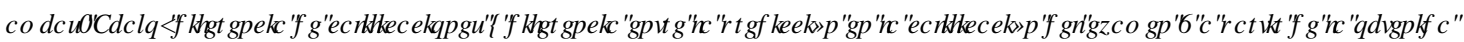

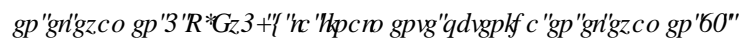

Un análisis ANOVA de las diferencias, o semidiferencias, observadas entre el primer y el último examen (exámenes 1 y 4 , respectivamente), para los diferentes cursos nos permite hacer el estudio de la diferencia entre estos exámenes de forma más clara, y poder discernir si hay efecto confinamiento. Efectivamente, existen diferencias significativas de éstas cuando se consideran los diferentes cursos ( $p=0,032$; ANOVA) Para determinar entre qué cursos se presentan las diferencias más notables, realizamos t-test entre pares de cursos, observándose solo diferencias significativas en las diferencias entre calificaciones del examen 1 y 4 al comparar los cursos 2017/18 y 2018/19 ( $\mathrm{p}=0,0014$; t-test). Este resultado indica que las diferencias no están relacionadas con la situación vivida en el curso 2019/20 y por tanto no se relacionan con el cambio de docencia ni el contexto de pandemia. De hecho, podemos entender mejor de donde viene esa diferencia realizando análisis ANOVA de las calificaciones de los exámenes 1 y 4 de los diferentes cursos. No se aprecian diferencias significativas en las calificaciones del examen 4 de los diferentes cursos como factor principal ( $\mathrm{p}=0,077$; ANOVA), pero sí para el examen $1(\mathrm{p}=0,00087$; ANOVA). Es más, el t-test entre cursos nos indica diferencias para las calificaciones del primer examen del curso 2017/18. con respecto a las de los cursos 2018/19 ( $\mathrm{p}=0,00077$ ) y 2019/20 ( $\mathrm{p}=0,00026$ ). Estos datos dan a entender que la posible anomalía se encuentre principalmente en las calificaciones del primer examen del curso 2017/18. 


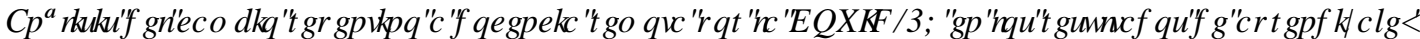

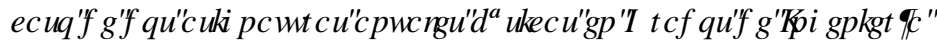

Del análisis estadístico realizado podemos concluir que no se aprecian diferencias significativas en las calificaciones de los estudiantes por el hecho de haber pasado de forma repentina de docencia presencial a remota debido a la pandemia por COVID-19. No obstante, cabe remarcar que este resultado no significa que no se hayan producido diferencias significativas en la docencia realizada, ni que estos no hayan influido en los resultados de aprendizaje, pero sí es un argumento a favor de una buena adaptabilidad de la docencia a la nueva situación, en la que se ha sabido acompasar el nivel de exigencia a los condicionantes del entorno, aspecto de gran importancia en la situación sobrevenida de crisis global y educativa, con un estrés evidente para todos los actores.

\subsection{Análisis de la asignatura de Matemáticas I}

En esta sección se hace el estudio de la asignatura de Matemáticas I del Grado de Ingeniería Electrónica y Automática. Se trata de un grupo reducido de estudiantes con Alto Rendimiento Académico, que es menos numeroso que los tratados en las secciones anteriores. No obstante, ambas asignaturas comparten aspectos semejantes tales como ser una asignatura básica anual de Grado de una Ingeniería.

En la tabla 4 se presentan los principales parámetros estadísticos de las calificaciones de los exámenes y sus combinaciones principales. En ellas, no se aprecia una diferencia clara para el curso 2019/20. Seguramente el aspecto más destacable sea un menor coeficiente de correlación $(0,29)$ entre el examen primero y último para el curso 2019/20 con respecto a los cursos anteriores que presentan valores bastante mayores. Aunque pueda ser razonable que las diferencias en metodología y evaluación disminuyan la correlación, la limitación de la muestra y del análisis estadístico hace difícil la obtención de conclusiones generalizables. De hecho, solo los cursos 2018/19 y 2017/18 presentan correlaciones Pearson significativas $(\mathrm{p}=0,012$ y $\mathrm{p}=0,020$, respectivamente).

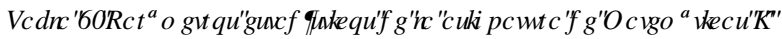

\begin{tabular}{ccccc}
\hline Magnitud & $\mathbf{2 0 1 9 / 2 0}$ & $\mathbf{2 0 1 8 / 1 9}$ & $\mathbf{2 0 1 7 / 1 8}$ & $\mathbf{2 0 1 6 / 1 7}$ \\
\hline N & 20 & 19 & 20 & 18 \\
Media Examen 1 & 7,0 & 7,8 & 5,4 & 6,1 \\
Desv. Estándar Ex1 & 1,4 & 2,2 & 2,4 & 1,8 \\
Media Examen 4 & 7,4 & 7,0 & 6,1 & 5,2 \\
Desv. Estándar Ex4 & 1,6 & 2,2 & 2,9 & 1,9 \\
Coef. Correlación & 0,29 & $0,58^{*}$ & $0,52^{*}$ & 0,37 \\
(* indica p $<0.05)$ & & & & \\
Media Semisuma & 7,2 & 7,2 & 5,7 & 5,7 \\
Desv. Est. Semisuma & 1,2 & 2,2 & 2,3 & 1,5 \\
Media Semidiferencia & $-0,22$ & 0,62 & $-0,36$ & 0,45 \\
Desv. Est. Semidif. & 0,89 & 1,2 & 1,3 & 1,0 \\
\hline
\end{tabular}

Un estudio ANOVA de las calificaciones de los diferentes exámenes, y de las diferencias entre examen primero y último, para los diferentes cursos nos indica diferencias significativas $(\mathrm{p}<0,05)$ para cada uno de estos aspectos $(\mathrm{p}=0,0018$ para examen $1, \mathrm{p}=0,037$ para examen $4, y \mathrm{p}=0,017$ para las diferencias entre exámenes). Realizando t-tests de las diferentes combinaciones, observamos que para el examen cuatro sólo 
presenta una combinación de cursos diferencias significativas: el curso 2019/20 con respecto al 2016/17, eso sí con un valor $\mathrm{p}$ destacable $(\mathrm{p}=0,00052)$. En el examen 1 , hay tres combinaciones de cursos con diferencias significativas: curso 2018/19 con 2016/17 ( $p=0,014$ ), curso 2019/20 con 2017/18 ( $p=0,016$ ) y curso 2018/19 con 2017/18 ( $\mathrm{p}=0,0019)$. Y si analizamos directamente la diferencia de calificaciones entre los dos exámenes del curso, cuatro combinaciones muestran diferencias significativas, aunque con valores p más modestos: curso 2018/19 con 2017/18 ( $\mathrm{p}=0,019)$, curso 2019/20 con 2018/19 ( $\mathrm{p}=0,018$ ), curso 2019/20 con 2016/17 ( $\mathrm{p}=0,041$ ), y curso 2017/18 con 2016/17 ( $\mathrm{p}=0,041$ ). Por tanto, podemos discernir que hay bastante variabilidad, pero que no se puede inferir que el efecto de la pandemia y confinamiento en el curso 2019/20 haya tenido un efecto destacable en estas variaciones.

\section{Conclusiones}

Con el fin de determinar si se observan diferencias en el grado de consecución de los objetivos de aprendizaje achacables a la situación de pandemia y confinamiento que impuso un cambio radical de docencia presencial a remota durante el curso 2019/20, se ha realizado un análisis de las calificaciones obtenidas durante dicho curso comparándolo con las de los cursos anteriores en dos asignaturas anuales básicas de dos Grados de Ingenierías. Se ha hecho especial hincapié en el análisis del primer examen (totalmente presencial) con el último (totalmente virtual en el curso 2019/20). Del análisis podemos concluir que no se aprecian diferencias significativas en las calificaciones de los estudiantes por el hecho de haber pasado de forma súbita de docencia presencial a remota debido a la pandemia por COVID-19. Si bien esto no asegura que no haya habido cambios en los resultados de aprendizaje sí es un argumento a favor de una buena adaptabilidad de la docencia a la nueva situación, en la que se ha sabido acompasar el nivel de exigencia a los condicionantes del entorno en situación de crisis sobrevenida.

\section{Agradecimientos}

S. Ardid agradece el apoyo del Programa CIDEGENT de la Generalitat Valenciana, CIDEGENT/2019/043.

\section{Referencias}

ARDID, M.; GÓMEZ-TEJEDOR, J. A.; MESEGUER-DUEÑAS, J. M.; RIERA, J. y VIDAURRE, A. (2015). “Online

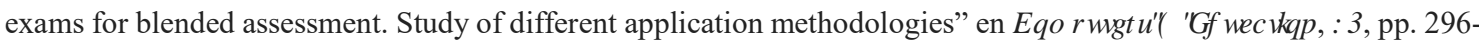
303.

ARDID, M.; MÁRQUEZ, S. y ARDID, M. (2021). "Use of sound recordings and analysis for physics lab practices"

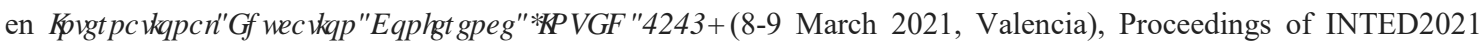
Conference pp. 7687-7693.

Salva Ardid1,2, Susanna Marquez3, Miguel Ardid1

ARISTOVNIK, A.; KERŽIČ, D.; RAVŠELJ, D.; TOMAŽEVIČ, N. y UMEK, L. (2020). "Impacts of the COVID-19 Pandemic on Life of Higher Education Students: A Global Perspective" en 6XWWQDEICWW QT, 8438.

GARCÍA-PEÑALVO, F.J.; CORELL, A.; ABELLA-GARCÍA, V. y GRANDE, M. (2020). "La evaluación online en la educación superior en tiempos de la COVID-19" en ( GXFDURQIQUKHL. QRZ OGJH6RFHH, 21, 12.

MAREK, MICHAEL W.; CHIOU SHENG CHEW; Y WEN-CHI VIVIAN WU. (2021). "Teacher experiences in converting classes to distance learning in the COVID-19 pandemic" en , QUHQDMRQDO-RXLODORI' LWDOFH( GXFDMRQ 7HFKQRQJIHW, -' ( 70 19.1, pp. 40-60. 
MOORHOUSE B.L. (2020) "Adaptations to a face-to-face initial teacher education course 'forced' online due to the COVID-19 pandemic" en - RXLDDORIC GXFDMRQIRU7HFFIQJ, 46:4, pp. 609-611.

RAPANTA, C.; BOTTURI, L.; GOODYEAR, P.; HWDO (2020). "Online University Teaching During and After the Covid-19 Crisis: Refocusing Teacher Presence and Learning Activity” en 3 RWUJLDD6FHQFH( GXFDURQ2, pp. 923945 . 